\title{
A perspectiva cognitivo-comportamental dos aspectos psicossociais que interferem na qualidade da relação médico-paciente
}

The cognitive-behavioral perspective of psychosocial aspects that interfere in the doctorpatient relationship quality

La perspectiva cognitivo-conductual de los aspectos psicosociales que afectan la calidad de la relación médico-paciente

\author{
Janaina Bianca Barletta* \\ Marcelo de Souza Gennari** \\ Rosana Cipolotti**
}

\begin{abstract}
Resumo
Este artigo tem por objetivo abordar os aspectos psicossociais que mediam a qualidade da relação médico-paciente com base em uma concepção cognitivo-comportamental. Para tanto, é feita uma breve revisão sobre os aspectos da relação de ajuda. Ressalta-se a importância da qualidade da relação estabelecida, destacando a participação de ambos na tomada de decisão e a relação colaborativa, empática e bidirecional. Assim, as crenças, necessidades específicas e características pessoais ganham lugar de destaque. Além disso, a organização empregadora, a equipe de saúde, o meio social e os familiares vão interferir na dinâmica da relação. $\mathrm{O}$ suporte social e a comunicação terapêutica são outros componentes da multifatorialidade desse processo. A relação positiva permite $o$ aparecimento de fatores de predisposição da doença, facilita o treino de habilidades assertivas de enfrentamento, a reestruturação cognitiva e a reeducação emocional, resultando na construção de respostas adaptativas a situações adversas.
\end{abstract}

Palavras-chave: Perspectiva cognitivo-comportamental, aspectos psicossociais, qualidade, relação médico-paciente.

\footnotetext{
Texto recebido em março de 2011 e aprovado para publicação em setembro de 2011.

Doutoranda em Ciências da Saúde pela Universidade Federal de Sergipe, mestra em Psicologia pela Universidade de Brasília, especialista em Psicologia Clínica da Saúde pela Universidade de Brasília, especialista em Terapia CognitivoComportamental pela Faculdade de Ciências Médicas de Minas Gerais, psicóloga. E-mail: jbar@terra.com.br.

** Médico, mestre em Ciências da Saúde pela Universidade Federal de Sergipe, especialista em Medicina do Esporte pela Unifesp.

*** Médica, pós-doutora na Universidade de Liverpool, doutora em Oncologia Pediátrica e mestra em Medicina pela Universidade São Paulo/Ribeirão Preto, professora adjunta da Universidade Federal de Sergipe.

**** Endereço para envio de correspondência: Janaína Bianca Barletta - Rua François Hoald, 263, casa 1 - Bairro Atalaia, Aracaju-SE. CEP: 49037-000. E-mail: jbar@terra.com.br.
} 


\section{Abstract}

This article aims to approach the psychosocial aspects that interfere in the quality of the doctor-patient relationships from a cognitive-behavioral design. For this purpose a brief revision on the aspects of the aid relationship is done. The importance of the relationship quality is highlighted, emphasizing the participation of both in decision-making and the collaborative, emphatic and bidirectional relationship. Thus, the specific beliefs, needs and personal characteristics gain prominence. Moreover, the employer organization, health team, social environment and relatives interfere in the dynamics of the relationship. The social support and therapeutical communication are other components of the various factors of this process. The positive relationship allows the bias factors of illness predisposition, facilitates assertive coping skills training, cognitive reorganization and emotional re-education resulting in the construction of adaptive responses to adverse situations.

Keywords: Cognitive-behavioral perspective, psychosocial aspects, quality, doctor-patient relationships.

\section{Resumen}

Este artículo tiene el objetivo de hablar acerque de los aspectos psicosociales que medían la calidad de la relación médico-paciente, desde un foco cognitivo-comportamental. Fue hecha una breve revisión sobre los aspectos de la relación de ayuda. La importancia de la calidad de la relación establecida tiene destaque, sobretodo la participación de ambos en la toma de decisión y la relación colaborativa, empática y bidireccional. Así, la creencia específica, las necesidades y los personales característicos ganan el lugar de prominencia. Por otra parte, el patrón de la organización, el equipo de la salud, el ambiente social y los familiares van a intervenir con la dinámica de la relación. La ayuda social y la comunicación terapéutica son otros componentes del multifatorialidad de este proceso. La relación positiva permite la observación de los factores de predisposición de la enfermedad, facilita los entrenamientos de capacidades asertivas de la confrontación, la reorganización cognitiva y la re-educación emocional dando por resultado la construcción de respuestas adaptativas a las situaciones adversas.

Palabras clave: Perspectiva cognitivo-conductual, aspectos psicosociales, calidad, relación médico-paciente. 
$\mathrm{N}$ o desenvolvimento histórico da medicina, algumas fases podem ser citadas, como a medicina espontânea, oracular, farmacopeica, hipocrática, medieval, moderna e contemporânea (Salgado, Salgado \& Salgado, 2002), e seu estudo aponta de que forma a preocupação com o cuidado da saúde foi se modificando. Inicialmente, quem tinha o poder da cura eram os xamãs e sacerdotes, ligando o trabalho com saúde ao místico e à arte. Essa medicina foi se transformando de maneira rápida, mas, durante muitos anos, a relação médico-paciente e o sigilo profissional foram os dois pilares que davam suporte para essa prática (Assad, 1993).

A partir do século XIX, a medicina tomou outra roupagem com as contribuições da biologia e da microbiologia na descoberta de microorganismos e, por conseguinte, nas causas de doenças, o que corroborou com o zeitgeist científico da época. Dessa forma, o cuidado com a saúde começou a ser calcado nos aspectos técnicos e nos avanços tecnológicos. Segundo alguns autores, essa nova forma de atuar em saúde afastou o médico do sentido humano e da importância da relação estabelecida com o paciente (Barletta, 2008; Ogden, 2004; Rodríguez, Kolling \& Mesquida, 2007).

Atualmente, a relação médico-paciente tem sido alvo de vários olhares e sua importância tem sido reconsiderada no processo saúdedoença. Não se questiona sobre os importantes progressos da ciência no tratamento de diversas doenças, mas nem sempre tais avanços garantem a satisfação do paciente com o atendimento, seu retorno às consultas, sua adesão ao tratamento e a emissão de comportamentos promocionais de saúde. Verifica-se que, além da importância da qualificação técnica, no atendimento em saúde, é necessário responder a outras demandas, principalmente de inter-relacionamento. Em geral, o paciente não sabe avaliar a competência do profissional, especialmente no início do acompanhamento, mas consegue fazer uma avaliação imediata da maneira com que foi atendido e cuidado (Pinheiro, 2005). Segundo Franco e Alves (2005), a satisfação do paciente está associada à quantidade de informação recebida na consulta, à comunicação social, à competência técnica e interpessoal. Esses aspectos fortalecem a confiança que o paciente credita ao médico (Robertson, Dixon \& Legrand, 2008).

A competência profissional está diretamente ligada ao conceito de qualificação, sendo necessário um conjunto de saberes para que uma tarefa profissional seja bem desempenhada em um contexto específico (Dultra \& Bastos, 2009). Para tanto, três dimensões sustentam o 
desenvolvimento da competência: conhecimento ou capacidade analítica, ligada ao domínio teórico; habilidade prática ou capacidade instrumental, ligada ao domínio técnico; e a habilidade social ou a capacidade de interrelação, ligada à capacidade de empatia, assertividade, comunicação, maturidade pessoal e condução ética (Cruz \& Schultz, 2009; Del Prette $\&$ Del Prette, 2003; Löhr \& Silvares, 2006).

Outro motivo que tem levantado a discussão sobre esse tema é o fato da grande preocupação sobre o processo de humanização, isto é, do resgate da dimensão humana na assistência. Assim, a importância do acolhimento, a intimidade e a naturalidade nas relações de ajuda devem andar de mãos dadas ao conhecimento científico, a fim de resgatar a saúde e a dignidade do paciente, funções primárias do atendimento médico (Crepaldi, 1999; Ezequiel et al., 2008).

Este artigo tem por objetivo abordar teoricamente questôes pertinentes a essa relação, com base nas contribuições do enfoque cognitivocomportamental, ressaltando os aspectos psicossociais presentes nesse contexto que mediam a qualidade da relação.

\section{Processo saúde-doença e seus mediadores}

Conforme o processo saúde-doença começou a levar em consideração outros aspectos além dos orgânicos, como os aspectos psicológicos e sociais (Ribeiro, 2007), a relação terapêutica novamente se tornou eixo fundamental das ações em saúde. Assim, aspectos ligados ao comportamento de promoção de saúde se tornaram essenciais. Para tanto, destaca-se a participação do médico e do paciente na tomada de decisão sobre o melhor tratamento possível, baseada na relação colaborativa, empática e bidirecional. Dessa forma, os aspectos pessoais, como crenças, habilidades comportamentais e características individuais específicas ganharam lugar de destaque sem desconsiderar aspectos do ambiente que influenciam na dinâmica da relação como a organização empregatícia, a equipe de saúde, o meio social e o familiar. Apesar dessa constatação cada vez mais evidente, segundo Malagris (1999), os profissionais de saúde não apresentam uma relação satisfatória com esses aspectos, limitando-se à sua prática técnica específica.

Uma das explicações para tal dificuldade encontra-se na diferença do repertório comportamental exigido nos modelos biomédico e biopsicossocial de saúde. Enquanto, no primeiro modelo citado, o médico era tido como o detentor absoluto do saber e seu foco de atenção estava 
voltado apenas para a doença, o segundo modelo prevê uma relação de saber mais equilibrada, a qual coloca o médico, a equipe de saúde e o paciente como corresponsáveis pelo desenvolvimento e manutenção da saúde. Dessa forma, o foco de atenção perpassa por todas as possíveis variáveis que possam interferir no adoecimento e não apenas nas questôes biológicas (Barletta, 2008; Neves Neto, 2004).

É nesse contexto que a Psicologia da Saúde foi sendo consolidada como uma interface importante da área. Uma das abordagens psicológicas que oferece explicaçóes, bem como estratégias de solução de problemas, é a cognitivo-comportamental (Barletta, 2003). Nessa perspectiva, a interpretação que a pessoa faz do adoecimento interfere na forma como ela lidará com o evento. Portanto o filtro para processar as informações e experiências, codificá-las e avaliá-las está baseado no sistema de crenças que o indivíduo desenvolve ao longo da vida. Dessa forma, o entendimento e a significação do problema vão influenciar na forma de enfrentá-lo, nas emoções sentidas e nas reações corporais, o que, por sua vez, também interferirão na avaliação cognitiva da situação (Malagris, 1999; Ogden, 2004). Ressalta-se que esse funcionamento é uma via de mão dupla em uma cadeia circular interligada com a interdependência entre cognição, afeto, fisiologia e comportamento. Além disso, está diretamente ligado ao contexto e à história de vida da pessoa.

Logo, quando o sistema de crenças está disfuncional, a percepção do ambiente ocorre de uma forma distorcida que, por sua vez, favorece a emissão do comportamento inadequado. Uma vez que se entende que toda e qualquer enfermidade acarreta uma série de desconfortos, com maior ou menor intensidade, gerando consequências no bem-estar do paciente, inevitavelmente interfere na resposta comportamental de promoção e prevenção de saúde do indivíduo. Esses comportamentos podem ser mais ou menos adaptativos, promovendo risco ou proteção ao organismo. Essa é apenas uma das formas que os processos psicológicos e comportamentais podem influenciar a saúde. Esses comportamentos, associados à doença ou à possibilidade de se ter uma doença, vão influenciar no processo de saúde. Assim, comportamentos e reações referentes à descoberta da doença, interpretação dos sintomas, tomada de decisão sobre a terapêutica e a adesão à prescrição médica vão mediar o prognóstico de saúde (Barletta, 2008; Baum \& Posluszny, 1999; Ogden, 2004; Ribeiro, 2007).

Os modelos de cognição social ajudam a explicar comportamentos de prevenção e promoção de saúde (Rutter \& Quine, 2002), uma vez 
que eles apontam como os processos cognitivos promovem a mudança comportamental, especialmente por meio da alteração no senso de autoeficácia (Bandura, Adams \& Beyer, 1977). Assim, conforme o indivíduo se percebe mais vulnerável a contrair uma doença ou quanto mais grave a percebe, mais ameaçadora e aversiva essa situação tornarse-á, o que, por sua vez, diminui a confiança na própria capacidade de lidar com a situação. A revisão feita por Barletta (2010) aponta que um maior senso de autoeficácia promove a percepção da capacidade para lidar com as adversidades, aumenta a motivação pessoal, potencializa desempenho e comportamentos resilientes.

O modelo de crenças em saúde foi desenvolvido a partir do início da década de 1950 por psicólogos sociais dos Estados Unidos, mas ficou conhecido com as publicações de Rosenstock na década de 1970 e hoje é considerado um dos modelos psicossociais mais importantes da área (Dela Coleta, 2003). O objetivo do modelo é explicar comportamentos preventivos, ou seja, não é um modelo de reestruturação de crenças, mas é útil para estudar os comportamentos frente aos sintomas (Dela Coleta, 2010) e nortear caminhos para intervençôes. Ele é baseado em quatro principais dimensões, sendo estas: (a) a percepção da pessoa em ser susceptível em contrair uma doença; (b) a avaliação da gravidade da doença com base nas crenças e emoções pessoais e das consequências como dor, morte, mudança no cotidiano e nas relaçôes familiares; (c) a percepção de benefícios que são referentes tanto às crenças de efetividade da ação quanto às consequências consideradas positivas; e (d) percepção negativa da ação promocional, preventiva ou mantenedora de saúde, considerada como barreira. As variáveis demográficas, psicossociais, estruturais e os estímulos para ação mediam as quatro dimensóes, que, por sua vez, influenciam a tomada de decisão, favorecendo ou minimizando a realização da ação promotora de saúde (Barletta, 2010; Dela Coleta, 2004; Nejad, Wertheim \& Greenwood, 2005).

De acordo com Malagris (1999), a forma com que a pessoa lida com a doença está diretamente ligada à forma como ela a percebe e a interpreta. Essa percepção é mediada por diversos fatores como a história de vida, experiências positivas e negativas com o adoecimento, modelos comportamentais e cognitivos aprendidos, além de vulnerabilidades pessoais e contingências ambientais. A reestruturação nessa percepção pode potencializar a emissão de comportamentos mais saudáveis. Como exemplo, os resultados da pesquisa de Faustino e Seidl (2010) com três pacientes soropositivos apontaram que os efeitos da intervenção 
cognitivo-comportamental são favoráveis para o aumento da adesão ao tratamento, da percepção da situação de saúde, do senso de autoeficácia, de estratégias de enfrentamento adequadas e do conhecimento sobre a doença, medicação e efeitos colaterais.

Pode-se dizer, ainda, que os aspectos emocionais, cognitivos e comportamentais também interferem de maneira direta na saúde, provocando mudanças biológicas do organismo. Estudos têm mostrado, por exemplo, que um aumento exagerado do nível de ansiedade pode elevar o risco de doenças, conforme aumenta a pressão na corrente sanguínea e acelera os batimentos cardíacos (Baum \& Posluszny, 1999; Ciechanowski, Walker, Katon \& Russo, 2002). Assim, o aspecto afetivo como sentimentos de medo, ansiedade, raiva, compaixão, entre outros, pode ser considerado como um fator central, pois, além de provocar tais efeitos no organismo, também proporciona mudanças comportamentais induzidas (Godoy, 1999; Jönsson, 2010). O estresse, por exemplo, pode levar o indivíduo a utilizar o álcool como estratégia para minimizar a aversividade da situação. De acordo com a revisão sistemática de Trigo, Teng e Hallak (2007), várias pesquisas têm relacionado o consumo de álcool e outras drogas ilícitas com o nível de estresse, especialmente no contexto de trabalho, levando ao burnout e à dependência química.

\section{A relação médico-paciente}

$\mathrm{O}$ aspecto relacional na abordagem cognitivo-comportamental é reconhecido como de extrema importância para o sucesso do trabalho terapêutico. Esse reconhecimento esbarra nas fronteiras do trabalho do psicólogo, atingindo a atuação de outros profissionais, inclusive do médico. Dessa forma, a relação médico-paciente pode ser chamada de terapêutica, uma vez que, por meio dela, podem-se maximizar efeitos positivos e minimizar efeitos negativos na construção da saúde.

Segundo Meyer e Vermes (2001), a relação terapêutica é uma importante variável que interfere diretamente no resultado do tratamento, podendo, inclusive, prever bons resultados e potencializálos. Essas autoras apontam que um dos fatores que levam ao fracasso do tratamento está relacionado com a negligência à qualidade da relação entre o profissional e o paciente, assim como a percepção do paciente sobre esse aspecto está ligada à efetividade do tratamento. De acordo com o modelo cognitivo, a forma como o indivíduo processa os eventos que ocorrem na sua vida influencia diretamente as suas respostas emocionais 
e comportamentais. Assim, a atribuição de significados ao evento/ situação atua como uma variável mediacional entre o real e as respostas do indivíduo, e para modificar as emoçôes e comportamentos (respostas), fazse necessária a intervenção sobre a forma de processar informações, isto é, sobre as cognições, esquemas e crenças dos pacientes.

A contribuição das diferentes variáveis envolvidas na geração e no prognóstico da doença vai determinar o tipo de tratamento e profissionais envolvidos. Para que a decisão mais adequada seja tomada, ambos os participantes do processo deverão interagir fazendo escolhas, traçando metas e desenvolvendo habilidades para solucionar problemas. Nesse sentido, tanto o médico quanto o paciente devem colaborar e assumir papéis de destaque. Uma relação terapêutica com qualidade faz com que médico e paciente se tornem parceiros, trabalhando com uma aliança sólida, favorecendo uma relação colaborativa.

Esse tipo de relação está ligado à troca recíproca entre seus participantes, porém uma dificuldade encontrada, de acordo com Franco e Alves (2005), está nos patamares diferenciados ocupados pelo médico e pelo paciente, o que dificulta o estabelecimento de um encontro positivo e favorece uma construção vertical e hierárquica. Ainda segundo esses autores, a consequência da pouca ou nenhuma concordância entre o pedido do paciente e a problemática apontada pelo médico é o adiamento das resoluções da demanda, assim como uma busca incessante do paciente por diversos profissionais, impedindo o estabelecimento de uma relação mais positiva.

Para entender essa importância, faz-se necessário pontuar os aspectos envolvidos diretamente nessa relação. Inicialmente, falava-se de uma relação unidirecional e assimétrica, na qual a redução do conceito de doença com minimização dos aspectos culturais, sociais e psicológicos, e a ênfase no tratamento farmacológico e na aparelhagem técnica moderna colocavam o paciente no papel de vítima da doença. Nesse sentido, o paciente, além de não ter controle, estava livre de qualquer responsabilidade sobre seu quadro clínico, o que diminuía a sua participação no processo saúde-doença.

Com uma nova noção sobre saúde, apontando para uma relação médicopaciente bidirecional e horizontal, outros componentes tornaram-se pontos fundamentais na dinâmica interpessoal. Tanto o médico quanto o paciente trazem consigo uma série de crenças, valores, necessidades específicas, características pessoais e sentimentos a respeito do outro, da situação e do que espera dela, conforme a figura 1 . 


\section{Figura 1}

Fatores que mediam a relação médico-paciente.

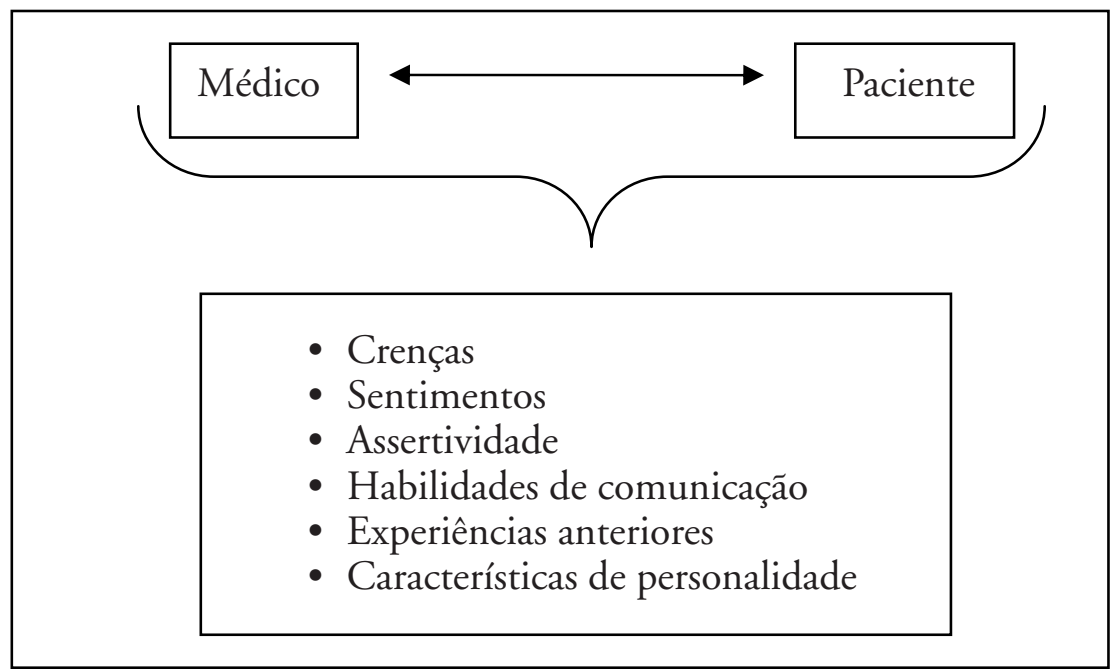

Algumas vezes, as crenças que o paciente tem a respeito da validade do trabalho médico e do tratamento são disfuncionais. Da mesma forma, o profissional credita comportamentos que o paciente deveria emitir, de acordo com suas crenças, que também podem ser distorcidas. Além disso, tanto o paciente quanto o médico, geralmente, repetem modelos antigos de relacionamento, já aprendidos e enraizados. Segundo Malagris (1999), os modelos cognitivos e comportamentais de pessoas significantes aprendidos desdea infância podem determinar respostas ao tratamento, uma vez que podem gerar reaçôes à doença e à figura do médico. Tais reações podem ser positivas ou negativas, intensificando ou minimizando as respostas promocionais de saúde. Pode-se dizer que se as crenças negativas que o paciente tem sobre si mesmo, sobre os outros e sobre o mundo são dominantes na sua percepção, então o comportamento tende a ser disfuncional e pouco adaptativo à situação. Da mesma forma, profissionais que mantêm expectativas irreais sobre os pacientes, em especial sobre aqueles que representam um desafio, podem ter dificuldades e reações desadaptativas em relação a estes (Beck, 2007; Ciechanowski et al., 2002).

Todos os aspectos biopsicossociais devem ser levados em consideração, uma vez que estarão norteando as ações dos participantes. Logo, para que a relação médico-paciente seja eficaz, é preciso harmonizar singularidades para chegar a um objetivo comum, o qual ambos estarão buscando. Tal postura pode minimizar os problemas e queixas do paciente. De acordo com esse raciocínio, tanto o médico quanto o paciente devem conhecer suas crenças e estratégias de lidar com elas. Quando um dos dois não as reconhece, 
crenças disfuncionais poderão interferir no estabelecimento de uma relação saudável. Isso ocorre porque as crenças podem provocar emoçōes intensas, aumentando a probabilidade de consequências negativas, como menor nível de tolerância em opiniōes contrárias, sentimento de frustração com o fracasso do tratamento, priorização de vulnerabilidades do outro em detrimento de potencialidades (Malagris, 1999; McMullin, 2005).

Durante um processo de enfermidade, as crenças, sentimentos e comportamentos só poderão aparecer ou terão mais facilidade em ser pontuados conforme a relação estabelecida com o médico for favorável. O médico, durante uma anamnese e no decorrer do atendimento, deve estar atento para construir um vínculo que permita uma troca efetiva de informações.

Quando se procura um atendimento de saúde, acredita-se que o médico e o paciente estão agindo de acordo com o mesmo objetivo, que é a procura pelo bem-estar do paciente. Porém, muitas vezes, esse bem-estar não tem o mesmo significado para o médico e para o próprio paciente. Enquanto o primeiro tem sempre a função de agir em busca da saúde física, muitas vezes a qualquer custo, para o segundo, o entendimento do bem-estar pode ser o menor dano, mesmo que isso implique a preservação do estado de doente. É pela relação médico-paciente que essas lacunas podem ser preenchidas e esclarecidas.

A relação médico-paciente positiva permite que a mediação cognitiva se torne clara para ambos os envolvidos. Por mediação cognitiva entendem-se as mudanças que os sentimentos e comportamentos provocam nos conceitos e imagens do indivíduo sobre a situação. Da mesma forma, a relação positiva permite que os ganhos primários e secundários, assim como as perdas primárias e secundárias que estão permeando o processo saúde-doença também possam se tornar evidentes (Kwee, 1999). Entende-se por ganhos os benefícios que o paciente recebe com a situação, enquanto a perda refere-se à tensão ou estresse que o paciente sofre. Da mesma forma, entende-se por primário os ganhos ou perdas diretamente relacionados à saúde, enquanto secundário está ligado aos ganhos ou perdas concretas além da saúde, como benefícios financeiros e esquiva de situaçôes aversivas.

Além do médico e do paciente, os familiares, a equipe de saúde, a instituição, o contexto social e ambiental também interferem na relação. É com base nos modelos familiares e sociais, no aprendizado anterior e em experiências sobre a doença que o indivíduo constrói suas crenças, pensamentos e comportamentos. As crenças populares sobre a medicação e a eficácia do tratamento, por exemplo, podem colocar em questão a importância 
e a necessidade de comportamentos promocionais de saúde (Barletta, 2003). Além disso, muitas condutas estão diretamente relacionadas com a expectativa que a organização empregadora tem do profissional. Dessa forma, a duração com que os atendimentos médicos ocorrem, assim como a urgência dos atendimentos, vai de acordo com a demanda dos hospitais e instituiçóes de saúde (Müller, 2009). O número de atendimentos é um dos parâmetros de produção para a quantidade de recursos inseridos naquela instituição, em especial organizações públicas de saúde, conforme a figura 2 .

Figura 2

Dinâmica da relação de saúde

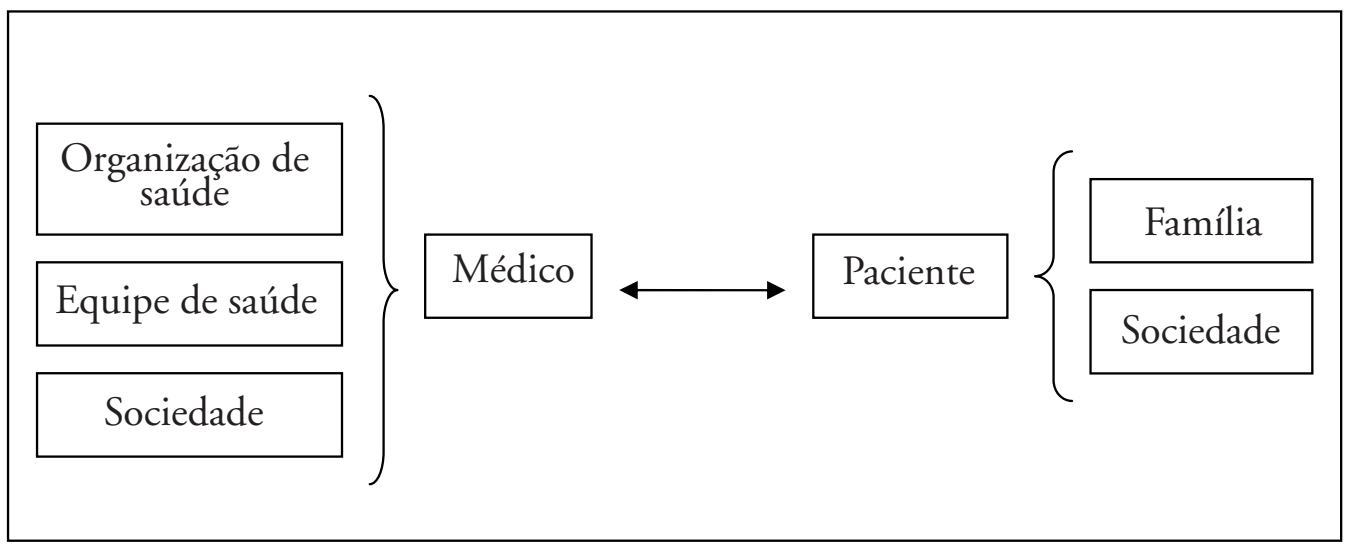

Outro componente importante da multifatorialidade que influencia a relação médico-paciente é o suporte social. Segundo Barletta (2003), a rede de suporte social, incluindo o apoio de amigos, familiares, equipe de saúde e do próprio médico, ajuda o paciente a dar continuidade aos comportamentos adaptativos e a lidar com os sentimentos estressantes como de medo, raiva, depressão e ansiedade. Auxilia-o, também, no enfrentamento assertivo e na relação de modo positivo com a situação.

Para que se estabeleça uma boa relação entre o profissional e o paciente, não se pode deixar de mencionar a comunicação como aporte fundamental (Almeida et al., 2005; Gask \& Upherwood, 2002). Esse aspecto é importante para consolidar a relação de confiança e estabelecer um vínculo positivo, tornando-se estratégia essencial para iniciar e manter um bom relacionamento entre o profissional e o paciente. Por sua vez, a relação médico-paciente, como um evento cíclico, pode minimizar os ruídos ou rompimentos que ocorrem na comunicação, em especial quando o sofrimento causado pelo estado de doença se torna 
insuportável. Vale ressaltar que a comunicação verbal é intensificada ou contraditada com base na comunicação não verbal, maximizando ou dificultando seu entendimento. Assim, a comunicação pode ter mais ou menos ruídos, a partir da maneira e do contexto em que ocorre, o que, somados aos conteúdos das informações prestadas influenciam nas interpretações do desenvolvimento da saúde (Franco \& Alves, 2005).

Com base em um nível adequado de comunicação, o canal fica aberto para ambos os participantes ouvirem, entenderem, discutirem, refletirem e construírem, em conjunto, habilidades e estratégias comportamentais de promoção de saúde, assim como sanar e manejar dúvidas, medos, expectativas e crenças distorcidas. Segundo Müller (2009), quanto melhor a qualidade da comunicação, maior facilidade de autorrevelação o paciente terá, aumentando a probabilidade de perguntas, minimizando o sofrimento e ansiedade e, consequentemente, maximizando a sensação de satisfação. Esse efeito se torna possível, uma vez que a função da comunicação vai além da pura e simples transmissão de informações. Sua função abrange também a necessidade de se fazer entender a mensagem, o que permite aos participantes do processo (profissional, paciente, familiares, equipe de saúde e organização) reverem conclusóes, reconstruírem respostas cognitivas e comportamentais, anteciparem possíveis fatos, influenciarem outras pessoas e ou eventos externos, compartilharem sentimentos, emoções e percepções (eventos internos), assim como iniciarem e modificarem processos fisiológicos corporais (Oliveira \& Gomes, 2004; Santos, 2001).

A assertividade é considerada uma habilidade necessária para o profissional de saúde e para o estabelecimento de uma comunicação terapêutica. Segundo Bolsoni-Silva (2002) e Alberti e Emmons (2008), quando o emissor, neste caso, o paciente, consegue expressar, de forma adequada, sentimentos e pensamentos para o médico (receptor), ele está emitindo um comportamento assertivo. O contrário também é válido. Assim, quando o médico consegue expressar-se de forma honesta, utilizando a entonação, latência e fluência de fala apropriadas, exercendo seus direitos, sem negar os direitos do outro, procurando atingir seus objetivos, sem prejudicar a relação com o paciente, está utilizando a assertividade. Além dessa característica, a empatia também é apontada como outro atributo importante para a relação médico-paciente, uma vez que possibilita a reflexão dos sentimentos trazidos pelo paciente, assim como possibilita a expressão de apoio por parte do profissional.

A comunicação, quando se torna terapêutica, permite o aumento da 
confiança, do respeito e da própria empatia entre profissional e paciente (Stefanelli, 2005). Esse fortalecimento da relação permite, por sua vez, que respostas adaptativas sejam mais facilmente alcançadas, como adesão ao tratamento, enfrentamento adequado e assertivo à situação de saúde, assim como maior probabilidade de mudanças no estilo de vida. Isso significa dizer que a comunicação terapêutica estabelecida na relação de saúde possibilita a implicação do paciente no cuidado e na responsabilidade pelo desenvolvimento de sua saúde. Como a participação direta e efetiva do paciente é pré-requisito para o bom andamento do processo, e essa participação tem maiores chances de se tornar possível com base em um vínculo positivo, a comunicação terapêutica passa a ser um dos pontos importantes da relação, pois permite que alguns problemas tenham maior probabilidade de serem contornados.

Portanto, a partir do exposto, reafirma-se que a aliança terapêutica firmada entre paciente e médico é um dos principais pilares que sustentam a relação de ajuda por meio de: a) informação facilitada sobre a doença, o tratamento e os efeitos colaterais, além do esclarecimento de dúvidas; b) motivação reforçada pelo médico por meio da comunicação verbal e da possibilidade de mudanças de atitudes negativas e prejudiciais ao tratamento, como efeito direto do modelo aprendido na relação com o profissional, assim como pelo resultado do tratamento; e c) aumento de habilidades comportamentais para lidar com o processo de adoecimento e seu tratamento, adaptando o estilo de vida individual aos comportamentos promocionais de saúde (Garcia, Lima, Gorender \& Badaró, 2005).

\section{Considerações finais}

A relação com o paciente vem ganhando importância na atuação em saúde, uma vez que se entende que tal aspecto é essencial para um bom prognóstico e para a promoção de comportamentos adaptativos. Nesse sentido, a relação médico-paciente passa a ser apontada como pilar fundamental para qualquer ato médico. Uma vez que a qualidade da relação terapêutica está diretamente relacionada à efetividade do tratamento no processo saúde-doença, verifica-se a necessidade de se pensar um pouco mais sobre ela.

A relação médico-paciente é permeada por diversos fatores e variáveis que vão interferir na resposta cognitiva e comportamental dos participantes no processo de saúde. É fundamental que tanto o médico quanto o paciente estejam cientes dessas variáveis, para que possam compreender a dinâmica do processo entre o indivíduo e o meio social e ambiental. Além disso, essa compreensão torna possível a busca de formas e estratégias de enfrentamento 
de situações difíceis que sejam beneficentes para ambos. Para tanto, é importante que o objetivo que media o processo saúde-doença esteja claro e que o seu estabelecimento seja comum para todos os envolvidos na relação de saúde.

Para que seja estabelecida uma relação positiva, deve-se estar atento a todas as variáveis que interferem no processo saúde-doença, em especial nas crenças distorcidas dos pacientes e nos comportamentos reforçadores dessas crenças. Geralmente tais comportamentos são de risco e irão mediar o desenvolvimento da doença.

A relação médico-paciente positiva permite o aparecimento de fatores de predisposição da doença, assim como facilita o treino de habilidades assertivas de enfrentamento, a comunicação terapêutica, a reestruturação cognitiva e a reeducação emocional, que são fundamentais para a construção de respostas adaptativas a situações aversivas.

\section{Referências}

Alberti, R. E. \& Emmons, M. L. (2008). Como se tornar mais confiante e assertivo. Rio de Janeiro: Sextante.

Almeida, H. O. et al. (2005). Desenvolvendo competências em comunicação: uma experiência com a medicina narrativa. Revista Brasileira de Educação Médica, 29 (3), 208-216.

Assad, J. E. (1993). Relação médico-paciente no final do século XX. In: Desafios éticos. (pp. 104-111). Brasília: Conselho Federal de Medicina.

Bandura, A., Adams, N. \& Beyer, J. (1977). Cognitive process mediating behavioral change. Journal of Personality and Social Psychology, 35 (3), 125139. Recuperado em 3 de novembro de 2010, de http://www.des.emory.edu/ mfp/Bandura1977JPSP.pdf.

Barletta J. B. (2010). Comportamentos e crenças em saúde: contribuições da Psicologia para a medicina comportamental. Revista de Psicologia da IMED, 2 (1), 307-317. Recuperado em 10 de outubro de 2011, de http://revistapsico. imed.edu.br/index.php/revistapsico/issue/view/7.

Barletta, J. B. (2008). Psicologia da Saúde e Medicina Comportamental: foco na atuação profissional. Cadernos de Psicologia da UFS, X (5), 19-37. 
Barletta, J. B. (2003). Treinando respostas de adesão ao tratamento de portadores de AIDS hospitalizados: um estudo exploratório. Dissertação de Mestrado, Curso de Pós-graduação em Psicologia, Universidade de Brasília, Brasília.

Baum \& Posluszny (1999). Health Psychology: mapping biobehavioral contribuitions to health and illness. Annual Review of Psychology, 50, 137163. Recuperado de 28 de fevereiro de 2002, de www.bellhowell.infolearning. com/pqdauto.

Beck, J. S. (2007). Terapia cognitiva para desafios clínicos: o que fazer quando o básico não funciona. Porto Alegre: Artmed.

Bolsoni-Silva, A. T. (2002). Habilidades sociais: breve análise da teoria e da prática à luz da análise do comportamento. Interação em Psicologia, 6 (2), 233-242.

Ciechanowski, P., Walker, E., Katon, W. \& Russo, J. (2002). Attachment theory: a model for health care utilization and somatization. Psychosomatic Medicine, 64, 660-667.

Crepaldi, M. A. (1999). Bioética e interdisciplinaridade: direitos de pacientes e acompanhantes na hospitalização. Recuperado em 22 de junho de 2007, de http://www.labsfac.ufsc.br/documentos.bioética.pdf.

Cruz, R. M., Schultz, V. (2009). Avaliação de competências profissionais e formação do psicólogo. Arquivos Brasileiros de Psicologia, 61 (3), 117-127.

Dela Coleta, M. F. (2003). Escalas para medida das crenças em saúde: construção e validação. Revista: Avaliação Psicológica, 2 (2), 111-122. Recuperado em 10 de outubro de 2011, de http://pepsic.bvsalud.org/pdf/ avp/v2n2/v2n2a03.pdf.

Dela Coleta, M. F. (2004). O modelo de crenças em saúde. In: Dela Coleta, M. F. (org.). Modelos para pesquisa e modificação de comportamentos de saúde. (pp. 27-62). Taubaté: Cabral.

Dela Coleta, M. F. (2010). Crenças sobre comportamentos de saúde e adesão à prevenção e ao controle de doenças cardiovasculares. Mudanças - Psicologia da Saúde, 18 (1-2), 69-78. Recuperado em 14 de outubro de 2011, de https:// www.metodista.br/revistas/revistas-ims/index.php.

Del Prette, A. \& Del Prette, Z. (2003). No contexto da travessia para o ambiente de trabalho: treinamento de habilidades sociais com universitários. 
Estudos de Psicologia, 8 (3), 413-420.

Dultra, J. A. L. \& Bastos, A. V. (2009). Trabalho, qualificação e competências: um desafio atual. RBDEPA - Revista Brasileira de Docência, Ensino e Pesquisa em Administração, 1 (2), 26-58.

Ezequiel, O. S. et al. (2008). Avaliação da abordagem do humanismo na relação médico-paciente, antes das mudanças curriculares e após, no curso de medicina da UFJF. HURevista, 34 (3), 167-172.

Faustino, Q. M. \& Seidl, E. M. F. (2010). Intervenção cognitivocomportamental e adesão ao tratamento de pessoas com HIV/Aids. Revista: Teoria e Pesquisa, 26 (1), 121-130.

Franco, A. L. S. \& Alves, V. S. (2005). A dimensão psicossocial e a autonomia do paciente: desafios para a relação médico-paciente no programa saúde da família. Psico, 36 (1), 99-106

Garcia, R., Lima, M. G., Gorender, M. \& Badaró, R. (2005). The importance of the doctor-patient relationship in adherence to HIV/AIDS treatment: a case report. The Brazilian Journal of Infectious Diseses, 9 (3), 251-256. Recuperado em 27 de agosto de 2007, de http://www.scielo.br/pdf/bjid/v9n3/a08v9n3.pdf.

Gask, L \& Upherwood, T. (2002). ABC of psychological medicine: the consultation. BMJ, 324, 1567-1569. Recuperado em 9 de setembro de 2007, de http://bmj.com/cgi/content/ful/324/7353/1567.

Godoy, J. F. (1999). Medicina comportamental. In: Caballo, V. E. (org.). Manual de técnicas de terapia e modificação do comportamento. (pp. 765-782). São Paulo: Santos.

Jönsson, B. (2010). Behavioural medicine perspectives for change and prediction of oral hygiene behaviour: development and evaluation of an individually tailored oral health education program. Doctoral Dissertation, Faculty of Medicine, Uppsala University, Swiss.

Kwee, M. G. (1999). Questôes sobre a terapia multimodal. In: Caballo, V. E. (org.). Manual de técnicas de terapia e modificação do comportamento. (pp. 629-661). São Paulo: Santos.

Löhr, S. S. \& Silvares, E. F. M. (2006). Clínica-escola: integração da formação acadêmica com as necessidades da comunidade. In: Silvares, E. F. M. (org.). Atendimento psicológico em clínicas-escolas. (pp. 11-22). Campinas: Alínea. 
Malagris, L. E. N. (1999). Relação Médico-paciente: uma visão cognitivocomportamental. Cadernos de Psicologia da SBP, 5 (1), 77-85.

McMullin, R. E. (2005). Manual de técnicas em terapia cognitiva. Porto Alegre: Artmed.

Meyer \& Vermes (2001). Relação terapêutica. In: Rangé, B. (org.). Psicoterapias cognitivo-comportamental: um diálogo com a psiquiatria. (pp. 101-110). Porto Alegre: Artmed.

Müller, M. R. (2009). Aspectos relevantes da comunicação em saúde. Psicologia IESB, 1, 72-79.

Nejad, L. M., Wertheim, E. H. \& Greenwood, K. M. (2005). Comparison of the health belief model and the theory of planned behaviour in the prediction of dieting and fasting behaviour. E-Journal of Applied Psychology: Social Section, 1 (1), 63-74. Recuperado em 5 de agosto de 2010, de http://ojs.lib.swin.edu. au/index.php/ejap/article/viewfile/7/16.

Neves Neto, A. R. (2004). Medicina Comportamental. In: Brandão, M. Z. S. (org.). Sobre comportamento e cognição. (v. 14). (p. 179-189). Santo André: Esetec.

Ogden, J. (2004). Psicologia da Saúde. (2. ed.). Lisboa: Climepsi.

Oliveira, V. Z. \& Gomes, W. B. (2004). Comunicação médico-paciente e adesão ao tratamento em adolescentes portadores de doenças orgânicas crônicas. Estudos em Psicologia, 9 (3), 459-469.

Pinheiro, L. M. H. (2005). A importância da Psicologia para a humanização hospitalar. Psicópio: Revista Virtual de Psicologia Hospitalar e da Saúde, 1 (1), 25-35. Recuperado em 15 de junho de 2007, de http://br.geocities.com/ revistavirtualpsicopio.

Ribeiro, J. L P. (2007). Introdução à Psicologia da saúde. (2. ed.). Lisboa: Quarteto.

Robertson, R., Dixon, A. \& Legrand, J. (2008). Patient choice in general practice: the implications of patient satisfaction surveys. Journal of Health Service, Research and Policy, 13 (2), 67-72.

Rodríguez, C. A., Kolling, M. G. \& Mesquida, P. (2007). Educação e saúde: um binômio que merece ser resgatado. Revista Brasileira de Educação Médica, 31 (1), 60-66. 
Rutter, D. \& Quine, L. (2002). Social cognition models and changing health behaviours. In: Rutter, D. \& Quine, L. (orgs.). Changing health behavior intervention and research with social cognition models. Great Britain: Biddles Limited, Guildford and King's Lynn. Recuperado em 18 de outubro de 2010, de www.openup.co.uk.

Salgado, J. V., Salgado, C. A. \& Salgado, J. A. (2002). A relação médicopaciente na história da medicina. In: Ferreira, E. C., Ribeiro, M. M. F. \& Leal, S. S. Clínica Médica - Relação Médico-Paciente, 2 (1). Recuperado em 20 de março de 2004, de http://www.bibliomed.com.br/book/showchptrs. cfm?bookid=143\&bookcatid $=15$.

Santos, A. C. A. (2001). Consulta médico-paciente: um estudo sobre aspectos comunicacionais da relação de cuidado em saúde. Dissertação de Mestrado, Curso de Pós-graduação em Psicologia, Universidade de Brasília, Brasília.

Stefanelli, M. C. (2005). Introdução à comunicação terapêutica. In: Stefanelli, M. C. \& Carvalho, E. C. (orgs.). A comunicação nos diferentes contextos da enfermagem. (pp. 62-72). Barueri: Manole.

Trigo, T. R., Teng, C. T. \& Hallak, J. E. C. (2007). Síndrome de burnout ou estafa profissional e os transtornos psiquiátricos. Revista de Psiquiatria Clínica, 34 (5), 223-233. Recuperado em 10 de outubro de 2011, de http:// www.scielo.br/pdf/rpc/v34n5/a04v34n5.pdf. 